factors on which Dr Daly suggests sample sizes should be calculated are arbitrary. The significance level and power should not be selected arbitrarily based on convention but be based on the clinical significance of obtaining false positive and false negative study results. This will then be a judgment based on relevance in real life rather than on statistical dogma.

Calculations of sample size also ignore biases that can be introduced by large studies simply because of the sheer practical difficulties in obtain ing a high participation or response. Similarly, as Rothman argues, sample sizes based on technical considerations alone fail to take into account the value of the information obtained. - It is important to consider what additional benefit there is to be gained for each increase in sample size. This will amount to an implicit marginal analysis of costs and benefits. Other practical issues of a sociocultural and political nature will also need to be considered.

One point from Dr Daly's paper illustrates the absence of clinical considerations. He has compared sample sizes based on the hypothesis testing approach and those based on the confidence interval approach and has shown in his example that a study based on the latter would have only a $50 \%$ power of detecting the corresponding smallest worthwhile differences. What about the largest worthwhile difference? To any physician or patient in despair it is the largest worthwhile difference that will inspire hope, and let us not underestimate the "power" of such hope.

Statistical consideration is only one of many in deciding sample sizes and must not be allowed to dominate such decisions or lend spurious scientific respectability. As Rothman summarises, "In the final analysis, the question of the most appropriate study size is not a technical decision to be determined by calculation but a judgment to be determined by experience, intuition, and insight" - and above all, by relating to the value of the information in bettering people's lives.

$$
\begin{array}{r}
\text { K M V NARAYAN } \\
\text { A BISSET } \\
\text { L MACDONALD }
\end{array}
$$

J CRESSWELI.

B WILSON

$\mathrm{V}$ J DEV

A CAIZOW

Department of Public Health Medicine.

Grampian Health Board,

Aberdeen $A B 9$ 8QP

1 Dalv LE. Confidence intervals and sample sizes: don't throw out ail your old sample size tables. B.117 1991;302:333-6. 9 all your old

2 Rothman KJ. Objectives of epidemiologic study design. In: Rothman KJ. Hodern epidemologv. Buston: Little, Brown. 1986.

SIR, - Many of Dr Leslie E Daly's arguments have been debated in medical and statistical journals but there is still much confusion and misunderstanding surrounding the problem of determining sample size in either a confidence interval or a hypothesis testing context.' 'The arguments put forward in favour of a hypothesis testing approach, however, may not be as convincing as the simple blood pressure example would have us believe.

Dr Daly suggests an example of a study in which a mean difference of $5 \mathrm{~mm} \mathrm{Hg}$ is observed with a confidence interval for that difference of $0 \mathrm{~mm} \mathrm{Hg}$ to $10 \mathrm{~mm} \mathrm{Hg}$. How much different is that information from the slightly different situation he describes of a mean difference of $4 \mathrm{~mm} \mathrm{Hg}$ with a confidence interval from $-1 \mathrm{~mm} \mathrm{Hg}$ to $9 \mathrm{~mm} \mathrm{Hg}$ ? One is "statistically significant" by some arbitrary cut off rule, the other is not. In practical terms, the data we have say either that one treatment is the same as the other $(0 \mathrm{~mm} \mathrm{Hg}$ and $-1 \mathrm{~mm} \mathrm{Hg}$ are in practice the same thing) or that one treatment may have a beneficial effect of up to about $9 \mathrm{~mm} \mathrm{Hg}$ or $10 \mathrm{~mm} \mathrm{Hg}$ (these two values also being trivially different). Dr Daly claims to determine sample size to fit in with estimation "with only a slight change of wording," though it is not the wording that matters but the concepts and objectives behind them. Dr Daly's objective would seem to be that of ensuring adequate power for a significance test.

Decision making rarely yields a simple yes or no answer $(p<0.05$ or $p \geqslant 0.05)$. Clinical trials and other comparative studies can rarely be assessed on the basis of a single efficacy parameter. We all strive to have as few efficacy parameters as possible, but the judgment of whether to prescribe (or register) any given treatment is a balance between how much good it might do, how much harm it might do, and, possibly, how much it costs. What should we ask if one treatment is significantly better than another but has significantly more side effects? Surely, "How much better and how much more?"

None of this is to say that significance testing is irrelevant. The size of an effect, however, is the crux of the problem, and appropriate clinical decisions may often be made in the absence of statistical significance but in the presence of sufficiently accurate estimates. Probably neither approach to sample size determination is appropriate to all studies, so I too would urge researchers to keep their old sample size tables and nomograms. They should not use them, however, without careful regard to the objectives of any particular study

Lilly Research Centre Limited,
Windlesham,

SIMON DAY

Surrey GU20 6PH

1 Daly LF. Confidence intervals and sample sizes: don't throw out all your sample size tables. B.MF 1991;302:333-6. (9 February.

AUTHOR'S REPLY, - The letters from Dr Narayan and colleagues and $\mathrm{Mr}$ Day raise some interesting issues relating to the place of sample size calculations in the planning of clinical research projects. I too think that it is wrong to base sample sizes solely on statistical criteria, and I would be the first to argue that the various parameters that go into such a calculation must be based on relevance to real life and sound clinical judgment. Mr Dav's comment on the necessity to take account of treatment costs and side effects in addition to treatment efficacy are also germane.

I fail to see, however, the relevance of "the largest worthwhile difference" mentioned by Dr Narayan and colleagues. Usually, sample size calculations are determined in a hypothesis testing framework and are based on a requirement that there be a good chance (the power) of detecting, with a statistically significant result, a treatment effect as large as or larger than a specified minimum worthwhile difference. Of course if the treatment effect is in reality much larger than this minimum the study will be much more likely to detect it. Sample size calculations, however, must be based on a realistic judgment of treatmen effects, and if these are set too high the study may in fact fail to detect worthwhile differences that are smaller. A study that misses a real effect can inspire no hope whatsoever - in either patient or physician

The main intention of my paper was in fact to warn against some recent methods of estimating sample size that seem clinically more relevant than the traditional techniques based on hypothesis testing but have serious shortcomings. The alternative methods, based on a confidence interval philosophy, suggest that the sample size be determined by prespecifying the width of confidence intervals (a measure of precision) without any consideration of power. I argued that such approaches paradoxically give unacceptably small study numbers and proposed a change of wording that would make the use of established methods compatible with a confidence interval analysis. $\mathrm{Mr}$ Day suggests that my arguments are not as convincing as thev might seem.

In my paper I used the example of a study to detect a blood pressure difference between treat ment and control groups of at least $5 \mathrm{~mm} \mathrm{Hg}$. A sample size of 251 in each group was determined on the basis of an $80 \%$ chance that the $95 \%$ confidence interval would exclude zero-equivalent to a $20 \%$ chance that zero would be included in the interval. Prespecifying the width of the $95 \%$ confidence interval as $10 \mathrm{~mm} \mathrm{Hg}$ resulted in the smaller figure of 123 -which gave a $50 \%$ chance of the interval including zero.

Mr Day seems to base his argument on the fact that including zero in the confidence interval does not really matter and that the interpretation of a confidence interval running from -1 to $9 \mathrm{~mm} \mathrm{Hg}$ is essentially the same as that of an interval running from 0 to $10 \mathrm{~mm} \mathrm{Hg}$. Although technically the former would be interpreted as a non-significant result, I agree that the two intervals are equivalent from the point of view of interpretation.

It is instructive, however, to examine the results that might be obtained from studies with the two different sample sizes if the true treatment effect were in fact $5 \mathrm{~mm} \mathrm{Hg}$. The smaller sample size gives a $34 \cdot 8 \%$ chance that a confidence interval resulting from the study would include $-1 \mathrm{~mm} \mathrm{Hg}$, whereas the corresponding chance with the larger sample size is only $8 \cdot 1 \%$. In more extreme situations the inadequacy of the smaller sample size becomes even clearer. The smaller study has a $16 \cdot 4 \%$ chance of ending up with a confidence interval including $-2.5 \mathrm{~mm} \mathrm{Hg}$ and an $11.9 \%$ chance of the interval including $-3 \mathrm{~mm} \mathrm{Hg}$. The corresponding figures with the larger sample size are $1.3 \%$ and $0.6 \%$. There is a considerable difference of interpretation between a confidence interval running from -3 to $7 \mathrm{~mm} \mathrm{Hg}$ and one running from 0 to $10 \mathrm{~mm} \mathrm{Hg}$, and such confidence intervals, compatible with the treatment increasing blood pressure by a clinically important amount, are quite likely with the smaller sample size. The smaller study could easily miss the treatment effect it was designed to detect.

Though it may not be the only criterion for determining the numbers required, a formal estimation of sample size is important in planning a clinical trial. It is vital, however, that such estimations are appropriate, and to this end I urge that we stay with the standard methods, reworded i necessary to fit into a confidence interval framework.

Department of Community Hedicine

LESLIE DALY

and Epidemiolog:

Liniversity (ollege D)ublis

D)ublin 2

Ireland

\section{Rectal examination in patients with abdominal pain}

SIR, - We would like to make three comments on the paper of $\mathrm{Mr} J \mathrm{M}$ Dixon and colleagues' from the viewpoint of the general practitioner. Firstly, there is no acknowledgment or discussion of the filtering effect of an initial assessment by a general practitioner on the sample of patients they studied. It is not clear from the paper how the sample of patients was obtained. We were informed by the authors that most patients had been seen and referred to the hospital by their general practitioner. The sample used in this study is therefore a highly selected one, and how it was obtained should have been stated.

Secondly, the authors have chosen to describe the value of the symptoms and signs by using odds ratios. This is only one of several methods that can be used to assess symptoms and signs, some of which will contribute different information and may be used in different situations to provide an overall picture of how useful a test is. For example, the specificity of a test gives an indication of how useful a test is at ruling out disease. By reworking 